\title{
Zwei Thomisten contra Kant.
}

\author{
Von Fritz Medicus in Halle a. 8.
}

I.

Franz Ludwig von Erthal, Furstbischof von Wtrzburg, hat bekanntlich seinen Professor der Philosophie, Matern Reuss, zu Kant nach Königsberg geschiokt, damit or dort von dem grossen Weisen selbst in die ganze Tiefe des nenen Systems eingefuhrt werde. Mehr als ein Jahrhundert ist seitdem vergangen. Auch in der Wtirzburger Diözese sind seit etwa 50 Jahren die Spuren des freien Erthal'schen Geistes verschwunden, und uberall in der katholischen Welt herrscht hente ein einziger Philosoph, Thomas von Aquino. „Sancti Thomae sapientiam restituatis et quam latissime propagetis" heisst es in der Encyclica ${ }_{n}$ Aeterni patris" vom 4. Ang. 1879. Wenn also auch meine folgenden Ausführungen nur Einzelfalle aufs Korn nehmen, so dürfen diese doch mit Recht als typisch betrachtet werden.

Schon mehrfach haben die "Kantstudien" Gelegenheit gehabt, daraut hinzuweisen, wie Thomas von Aqnino für die katholische Kirche von ahnlicher Bedeutung ist, wie Kant für den Protestantismus (vgl. Bd. I, Hft. 3 and 4, 8. 442, 479; Bd. II, Hft. 4, S. 485), und weil der Katholizismus weiss, wie eng Kant zum Protestantismus steht, richtet er seine Polemik gegen keinen anderen Philosophen mit nur annähernd ähnlicher Intensität wie gegen den Lehrer der Autonomie des sittlichen Bewusstseins, den Lehrer der protestantischen Sittlichkeit. - Aber man greift klug genug nicht gleich da an, wohin man hinaus will, sondern man wendet sich zunächst gegen die erkenntniskritische Grundlage der Kantischen Ethik, man sucht den stolzen Bau von Grund aus za zerstören, und wenn wir der "Re $R u \in$ Thomiste" glauben dürten, so wäre gute Aussicht da: „Sa base est fragile, ses ligatures n'ont que l'apparence de la solidité (Revae Thomiste, 5. Jahrg., Nr.1, S. 3). Genanntes Organ der Dominikaner brachte nämlich im vorigen Jahre in der Mărz-, Mai- und Septembernummer (Nr. 1, 2 and 4) eine Artikelserie mit der Tendenz, die - Inferioritat Kants gegentiber der Aristotelischen Scholastik darzathun. Die Aufsătze entstammen der Feder des Dominikanerpaters A. Gardeil. Den Anlass ihres Erscheinens bieten die Kantrorlesungen des - auch 
an der Redaktion der ${ }_{n}$ Kantstudien ${ }^{*}$ beteiligten - bekannten Pariser Professors E. Boutroux. (Diese Vorlesungen wurden bereits im ersten Band der „Kantstudien“, S. 491 angezeigt). Der dritte Artikel: „Ontils vraiment 'dépassé Kant'?" knüpft dann noch an Schriften dreier französischer kritischer Philosophen, Gory, Thouverez und Brunschvigg, an. Er ist für uns von geringerem Interesse. Seine Absicht ist, den genannten Denkern nachzuweisen, dass sie vergeblich streben, über Kant hinauszakommen, so lange sie an der kritischen Grundthese festhalten, dem Bruch $z$ wischen Vorstellungswelt and Sein. Wichtiger für uns schon ist die zweite Abhandlung: „Devons-nous 'traverser Kant'? " Boutroux hat in seinen Vorlesungen die Frage bejaht: Wenn wir über Kant hinausgehen wollen, müssen wir durch seine Kritik hindurchgehen, müssen wir sie "traverser d'un bout à l'antre" (Nr. 2, S. 180). Was heisst nun „traverser"? Gardeil sagt ganz richtig, es könne zweierlei bedeuten: entweder man geht durch eine Lehre hindurch wie durch eine unsichere Gegend, um sie kennen zu lernen and imstande zu sein, ihre Gefahren zu meiden. Dergleichen unangenehme Beschäftigungen seien oft recht nützlich, mitunter notwendig. Und in diesem Sinne sei es auch ganz gut, wenn man durch die Kantische Kritik hindurchgehe. Das sei aber nicht die Meinung Boutroux': Boutroux hat sein vorhin zitiertes Wort selber durch den Ausdruck umschrieben "es ist nicht mehr erlaubt, sich dem Kantischen Kritizismus zu entziehen.“ „Man muss durch ihn durchgehen" heisst also hier ${ }_{n}$ man muss ihn in sich aufnehmen ${ }^{*}$. Boutroux ist nun aber keiner von denen, die anf des Meisters Worte schwören; er hat an den Resultaten der Kantischen transscendentalen Aesthetik and Analytik mannigfache Kritik geübt. Er will ja selbst "dépasser Kant". Was ist es also, dem er sich „nicht entziehen" kann? Nun, erstens bleibt auch von den positiven Ergebnissen der Kantischen Lehre noch gar Manches tbrig; dann aber vor allem der Geist der kritischen Forschung: die Methode. Das ist die Antwort, die man erwarten sollte, die aber Gardeil nicht giebt. Die Uebereinstimmung in den positiven Resultaten ignoriert er, und uber die Methode Kants hat er bereits im ersten Artikel eine Ansicht entwickelt, auf die wir noch za reden kommen. Nach Gardeils Meinung bleibt ron Kants Werk nur tbrig „la partie destructive et négative" (181) als dasjenige, das Boutronx annehmen darf. Nun aber steht für unsern Scholastiker die Sache so: Der negative Teil der Kantischen Kritik ist eine Demonstration der Unmöglichkeit der Metaphysik. Wenn damit anch die Aristotelisch-scholastische Metaphysik bekämpft sein sollte, dann wäre es allerdings nötig, in Boutroux' Sinne durch die Kritik hindurchzugehen, um tber sie hinauszakommen. Diese Voraussetzung trifft jedoch nach Gardeil nicht za: Die Lehre von der intellektnellen Anschaung, wie sie die klassische Metaphysik der Scholastik hat, wird nicht berührt von Kants Argumentationen, die sich nar gegen den ontologischen Dogmatismns kehren. Diejenige intellektuelle Anschanung, deren Ueberwindung durch Kant Boutroux verktindet hat, ist die Lehre der Platoniker, dass wir in unmittelbare Beziehnngen za den Ideen za treten vermöchten (183)- unerschtittert aber steht noch die Aristotelische Lehro von der intellektuellen Anschanung, 
d.r Erkenntnisweise, die ausgeht von der Erfahrung und durch verstandesmässige Abstraktion die Objekte für die intellektuelle Anschaung präpariert. Mit schwer verbissenem Grimm wird Boutroux vorgeworfen, dass er die Hauptsache unberllaksichtigt lasse: ${ }_{n}$ Ponrquoi donc, dans cette seizième leģon, où il détermine le caractère intuitif des principales doctrines dogmatiques, M. Boutroux cite-t-il Platon, Descartes, Leibnitz, et passe-t-il sous silence, je ne dis pas les scolastiques (on sait qu'ils ne comptent pas), mais Aristote? Pourquoi nous laisser penser qu'il enveloppe la méthode du Stagirite et de ses disciples dans le reproche de $\pi \rho \tilde{c} \tau 0 \nu$ $\psi \varepsilon v \tilde{\delta} \sigma_{S}$ dont l'intuition intellectuelle est frappée? S'il se fat sourena d'Aristote, n'aurait-il pas dâ examiner, entre l'intuition directe des êtres transcendantanx et la conscience de l'être immanent, la possibilité d'une connaissance intuitive abstractive ? $^{4}$ (184) Damit ist der Stab tber die Kritik gebrochen: "Il n'est pas besoin de la traverser pour la dépasser" (185). - Bevor ich daran gehe, einige kritische Bemerkangen zu geben, will ich versuchen, den Kern dieses Gedankenganges in gelänfiger Terminologie anf einen Syllogismus za bringen, und da lantet er so: Nach Kant besteht die Metaphysik aus synthetischen Urteilen a priori, and nor eine solche Metaphysik wird von ihm widerlegt. Nun aber kennt die scholastische Metaphysik keine synthetischen Urteile a priori. Folglich wird sie von Kant nicht getroffen. - Man beachte wohl: Gardeil giebt zu, dass die Unmöglichkeit, eine intellektuelle Anschanung zu deduzieren, den Platonismus, der das Ansichseiende unvermittelt erfassen will, trifft (183); den scholastischen Begriff der intellektuellen Anschanung dagegen soll sie nicht treffen. Der Grund kann nur darin liegen, dass wohl die Platonische, nicht aber die Thomistische Metaphysik aus synthetischen Urteilen a priori besteht; denn die Scholastik geht von der Erfahrung aus. - Die Kritik der Gardeil'schen Antikritik kann sehr kurz sein: sie brancht nämlich nur auf Kants Widerlegung der Beweise für das Dasein Gottes za verweisen. Der kosmologische und der physiko-theologische Beweis gehen von der Erfahrung aus: sie beide scheinen aus synthetischen Urteilen a posteriori zusammengesetzt zn sein, and dass sie auch in der Scholastik so angesehen werden, davon kann sich Gardeil dadurch tuberzeugen, dass er sie in der Summa theologiae seines Ordensbruders nachschlägt, die bekanntlich keine synthetischen Urteile a priori enthalten soll; sie sind zn finden Tom. I, qu. 2, art. 3 . (in Migne's Ausgabe S. 622-624). Sodann mag er die Kritik der reinen Vernunft anfschlagen und nachsehen, ob nicht Kant doch gezeigt hat, wie gerade das beweiskräftige in diesen Demonstrationen stets in darin versteckten synthetischen Urteilen a priori liegt; damit aber erweist es sich, in Folge mangelnder Deduktionsmöglichkeit, als ein beweisankraftiges. ${ }^{1}$ ) Was nun von den Beweisen für das Dasein Gottes gilt,

1) Thomas hat gewiss darin Recht, dass der ontologische Beweis durch die anderen Beweise unterstlitzt werden muss, wenn er etwas beweisen soll (vgl. qu. 2, art. 1.); Kant hat aber nicht minder Recht, wenn er darauf hinweist; dass die beiden anderen (auf die sich die 5 a. a. 0 . von Thomas gebrachten reduzieren lassen) der Stutze des ersteren bedürfen. Das Verhältnis, in dem dis Beweise zu einander stehen, ist das jener Geschäftsleute, die erst gegenseitiz fuir 
gilt auch allgemein: die Metaphysik des doctoris angelici tiberschreitet unleugbar die Erfahrung; die Erfahrung aber ist das Gebiet and die Grenze der Erkenntnis. Was jenseits dieser Grenze liegt, ist, weil kein Organ, mittels dessen man seiner habhaft werden könnte, deduzierbar ist, verbotene Frucht; wer also etwas daruber anssagt, that es nach dem Satz vom unzareichenden Grund. - Betrachtet man den Schluss dieses Artikels, so kann man unschwer aus den gerade hier höchst bescheidenen Ausdrücken, den immer wiederholten Fragen ${ }^{1)}$ erkennen, wie wenig der Verfasser imstande war, eine positive Begründung zu geben. Er mag es selbst gefthlt haben, dass sie nur nach dem soeben genannten unlogischen Grundprinzip hätte geschehen können. Wenn er aber (193) Fausts Worte "Weh', steck' ich in dem Kerker ${ }^{2}$ ) noch ..." zitiert, so zeigt er damit darauf hin, dass alle Metaphysik ihren Grand hat in den Wünschen, die die Väter der Gedanken sind. Kant hat sehr wohl gesehen, dass je nach der Verschiedenheit der Wünsche die metaphysischen Systeme verschieden ausfallen, so dass der scholastischen Metaphysik eine ganze Reihe nicht minder "beweiskräftiger" Systeme entgegengesetzt werden kann.

Doch nun zum interessantesten der drei Artikel, dem ersten. Er ist tiberschrieben: "Après le cours de M. Boutroux." Der Titel will sagen, dass der Verfasser Boutroux' Vorlesungen studiert hat, und dass für ihn Kant seitdem ein anderer ist als vorher. Gardeil giebt sich den Anschein, dass er fruher die Kritik der reinen Vernunft für viel gefährlicher gehalten hätte. Nan aber hat er aus Boutroux' Cours gelernt, dass die eigenartige and für seine ganze Philosophie hochbedentsame Fragestellung Kants (sc. nicht nach dem nSein", sondern nach der "Möglichkeit der Wissenschaft und der Moral") ihre psychologische Wurzel habe einerseits im Studium Newtons, andererseits in der pietistischen Erziehnng - and nun erscheint ihm "la terrible Critique" (Nr. 1, S. 4.) nicht mehr unangreifbar. Denn ,elle a sa source subjective dans des conditions de miliea * (4). Boutroux hălt nun jene Fragestellang für verkehrt, da wir nicht berechtigt seien, die Existenz der Wissenschaft als Thatsache, von der aus man deduzieren könne, vorauszusetzen. Eine Prufung des Bontroux'schen Einwandes anzustellen, fällt Gardeil nattirlich nicht ein; davon, dass eine grosse Reihe hervorragender Kantforscher ganz anders ther die Kantische Fragestellng denkt (die auch ich fur verfehlt halten witde, wenn sie wirklich von dem Postalat der thatsächlichen Existenz wissenschaftlicher Erfahrung ausginge), erfährt der

einander bürgten, dann aber beide Bankrott erklärten. Die Rolle der Bank, bei der die edlen Herren ihre Kapitalien aufnahmen, wird heute von der Scholastik gespielt.

1) nIst denn wirklich der Aristotolische und Thomistische Realismus falsch? Sollte es nicht möglick sein, dass das intellektuelle Phänomen uns zaw transscendentalen Wesen führt? Ist denn notwendig die Wahrheit ein möglicher Irrtum, die Behsuptung im Urteil eine Illusion, die Philosophie im Grunde eine Mystifikstion?" (193) - Ich sehe hier davon ab, auf das gríndliche Missverständnis einzugehen, das in den letzten Worten enthsiten ist.

$\left.{ }^{2}\right)$ Auch hier sei abgesehen von dem gar nicht passenden Vergleich der Erscheinungswelt mit oinem Kerker. 
Leser nichts. Ueberall, wo Boutroux Kant angreift, hat er für Gardeil -eo ipso recht; andere Denker sind nur da und tberall da Autoritat gegen Bontroux, wo sie Kantische Thesen ablehnen, die Bontroux verficht. So muss z. B. gegentlber der Mathematik, deren Aprioritat Boutroux vertritt, die Metageometrie herhalten, um die Ḱantische Behauptung, dass es synthetische Urteile a priori giebt, za bestreiten (16). Denn es gilt anf alle Weise den Schlnss zu erhalten: „L'existence des principes synthetiques à priori dans la physique et les mathématiques est loin d'être démontrée clairement" (17).

Ich entziehe mich der undankbaren Aufgabe, Gardeil die zahlreichen Missverständnisse in Betreff der Grandlagen des Kantischen Kritizismus vorzuhalten; denn für einen Kundigen sind sie völlig durchsichtig, und wenn ihm selbst daran gelegen wäre, Kant zu verstehen, so hätte ihm die längst vorhandene Litteratar genug Hilfsmittel bieten können. Er scheint jedoch Kant selbst ubberhaupt nicht, and von Kantfreandlichen Schriften nur die Vorlesungen Boutroux' gelesen za haben. Dass man nun, wenn man zum ersten mal näheres über Kant hört, ihn nicht gleich richtig versteht, ist eine Erfahrung, die schon viele gemacht haben, und deren sich anch Gardeil nicht zu schämen braucht. Nur braucht man dann seine ersten Reflexionen über ein Thema von solcher Wichtigkeit and Schwierigkeit nicht sofort dracken za lassen. Znm Beweis dafür, dass nicht za viel gesagt ist, wenn Gardeils Elaboraten die erforderliche Abklärang abgesprochen wird, dürfte schon das bisher Ausgeführte genügen; noch dentlicher wird es jedoch werden bei Besprechung des Abschnitts im ersten Artikel, zu dem ich mich jetzt wende. Er trägt den Titel: „La méthode de Kant et la méthode scolastique" und beginnt mit der Behauptung, "que la méthode kantienne, telle qu'elle ressort de l'exposé de M. Boutroux, n'est nullement originale dans ses procédés, qu'elle les emprante presque tous à la scolastique, qu'elle ne se distingue de la méthode péripatéticienne que dans les détours qu'elle lui donne à l'intention de mystifier. cenx qui croiraient rencontrer dans la Critique la solution da problème de la Vérité (19)! Dass die folgende Ausführang dieses Themas nur bei denen verfangen kann, die Kant nicht kennen, ist von vornherein za erwarten. Aber ich will nicht so boshaft sein wie Gardeil und behaupten, der Abschnitt sei geschrieben "à l'intention de mystifier"; dann aber muss mir der Verfasser erlauben, ihm totale Verständnislosigkeit gegenüber der Kantischen Lehre vorzawerfen. Obwohl nun freilich für die Kenntnis Kants nicht das mindeste ans einer solchen Arbeit resultieren kann, hat es doch einiges Interesse, zu sehen, wie Gardeil seine kühne These begründet. Er spricht zunächst über das Prinzip der Methode. Dieses ist nun darum bei Kant and den Scholastikern identisch, weil beide das Gegebene nicht als letzte Thatsache hinnehmen, sondern es metaphysisch zu analysieren suchen. Zum Beweis wird auf einige Beispiele hingewiesen, in denen der heilige Thomas nach der analytischen Methode verfährt. Freilich muss dann zugegeben werden, dass es doch einen Unterschied giebt ${ }_{\eta}$ dans la manière de considérer le fait" : Kant fragt nach den Bedingungen der "blossen "Phänomene, die Scholastiker fragen nach denen der Dinge selbst. Jedenfalls lautet es nicht übel, wenn der 
Verfasser dieses Verhältnis so charakterisiert: „Les scolastiques pénètrent plus profond" (21). Dass die Verschiedenheit der Resultate aus dieser Höhendifferenz der beiderseitigen Standpunkte folgt (22), ist eine der wenigen richtigen Behauptungen Gardeils über Kant. Wenn jedoch dieser fundamentale methodologische Unterschied als eine solche Kleinigkeit behandelt wird, dass es noch immer erlaubt ist, Kants Methode mit der scholastischen zu identifizieren, so bleibt das so sehr auf der Oberfläche, dass es sich bloss dann lohnen würde, näher darauf einzugehen, wenn ich für den Leserkreis der "Revue Thomiste" schriebe. - Ganz amusant ist zu lesen, wie Kant nachgewiesen wird, dass er sich der von Aristoteles mustergiltig niedergelegten Arten der Demonstration zu bedienen pflegt, noch amusanter, dass selbst in der metaphysischen und der transscendentalen Deduktion unser Scholastiker alte Bekannte entdeckt, da nämlich auch seine Schule die quaestio facti und die quaestio iuris stellt (23). Er meint "die Scholastiker werden lächeln", wenn sie diese Verwandtschaft entdecken: ich glaube ihm die Versicherung geben zu dürfen, dass auch die Kantianer lächeln werden, wenn sie von dieser Verwandtschaft hören. - Freilich muss sich Kant, nachdem sein scholastischer Gegner prinzipielle Einigkeit konstatiert hat, von ihm eine recht stümperhafte Durchführung der an sich richtigen Prinzipien vorwerfen lassen. - Zum Schluss dieses Abschnittes kommt der Verfasser noch auf einen Punkt zu sprechen, in dem er wenigstens nicht ganz unrecht hat. Er sagt, dass sich eine Methode auch charakterisiert durch ihre résultats immédiats. Dahin rechnet er bei Kant die Unterscheidungen zwischen Materie und Form, zwischen Sinnlichkeit and Verstand, zwischen Phänomen und Ding an sich. Und nun kommt ein Satz, in dem Wahres und Falsches wunderlich gemischt sind: "C'est ici que se manifestent surtont les emprunts faits par Kant à l'école, et le manvais usage d'éléments très légitimes en soi pour une fin préconçue" (26). Wahr ist an dieser Behauptung, dass sich hier eine Nachwirkung scholastischer Einflüsse anf Kant zeigt; aber Kant war bei dieser Beeinflussung völlig passiv und hätte diese Verschnörkelungen durchaus nicht zu seinem System nötig gehabt; völlig falsch ist jedoch - und das hängt mit dem eben Gesagten zusammen - der zweite Teil des Satzes: in Kants System sind die scholastischen Momente ausscheidbare Nebensachen, in der Scholastik sind sie das Material der philosophischen Arbeit. Viele Gelehrte der Gegenwart sehen eine grosse und schöne Aufgabe darin, den hinter Kants scholastischen Formeln verborgenen Kern zu Tage zu fördern. Was aber übrig bliebe, wenn man mit der scholastischen Philosophie so verfahren wollte, darüber brauche ich keine Worte zu verlieren. Ich begnüge mich mit dem Hinweis darauf, dass der Neuthomismus ganz anders zu Thomas steht als der Neukantianismus zu Kant. - Das Grundmissverständnis, mit dem Gardeil an die Kantische Lehre herantritt, ist dieses: er sieht nicht, wie Kants neue Fragestellung den Begriff der Wissenschaft verändert, indem sie ihr ein anderes Objekt zuweist; er meint, der Erkenntnis sei ihr reales Objekt nun genommen, die Welt der Phänomene sei eine Welt der "Illusion ${ }^{\prime}$, die Sinnlichkeit sei bei Kant ein nobstacle de la connaissance ${ }^{\text {u }}$ (28). Vielleicht aber ist's ganz gut, dass er Kant darin missverstanden hat. Denn ich fürchte. er hätte sonst 
behanptet, Kant habe auch die Lehre, dass die Sinnlichkeit das erkenntnisvermittelude Prinzip sei, von der Scholastik geborgt, dann aber „manvais usage" davon gemacht. Ich bitte, letzteres nicht als einen blossen Scherz zu betrachten. Denn wenn dÆe bereits zitierten Stellen noch nicht genugen sollten, um za zeigen, wie Gardeil das Verhältnis der Kantischen Lehre zur Scholastik auffasst, so genügt daza gewiss die letzte, die ich noch anfuhren will: „La Critique n'est qu'an plagiat, et encore il est mal fait" (29). Dieser Satz braucht keinen Kommentar mehr; höchstens brauche ich eine Entschuldigung dafür, dass ich Gardeil zu ernst genommen habe.

II.

Bücher haben ihre Schicksale, und das Schicksal hat mitunter Anwandlungen von Ironie. Eine solche scheint es auch gehabt zu haben, als es im vorigen Jahro gleichzeitig mit des Würzburger Professors Schell Schrift "Der Katholizismus als Prinzip des Fortschritts" den dritten Band von Willmanns "Geschichte des Idealismus" hat erscheinen lassen. Schell schreibt für den deutschen Katholizismus; darum kann er auch nur durch ein deutsches Buch ad absurdum geführt werden. Gardeil ist keine Gegeninstanz gegen ihn; Willmann ist mehr als das: er liefert die Parodie. Gardeil ist Franzose, er ist Priester, er ist unbewandert in der Kantischen Philosophie, er ist in Folge seiner Stellang nicht imstande, alle die feinen Verästelungen des grossen Stammes: Geistesleben zu beobachten. Anders Willmann. Willmann ist Deutscher, er ist Laie, er hat die Kantische Philosophie studiert, er steht im Centrum der geistigen Bestrebungen der Gegenwart und hat längst durch seine Werke bewiesen, dass er ein feinsinniger Mann ist, dessen reicher Begabung kein Trieb jenes Stammes fremd zu bleiben braucht. Und darum ist er ernster zu nehmen als der ausländische Dominilsaner, er ist sehr ernst za nehmen. Sein Buch ist ein bedrohliches Zeichen der depravierenden Macht des Ultramontanismus. Ihm gegenüber macht Gardeil den Eindruck des unschuldigen Gemüts, das an seinem Teile mitarbeiten will an der Bekämpfung der Kantischen Inlehren, das in dieser Unschuld nach Argumenten sucht, mit denen es sie zu treffen und zu überwinden meint; Gardeil hält seine Argumente für neue, wertvolle Beiträge zur Apologetik. Ganz anders Willmann. Willmann muss wissen, dass das, was er gegen Kant sagt, längst schon von anderen gesagt ist, anch von Protestanten; was er bringt, ist nicht von jener originellen Verkehrtheit wie die Einfälle Gardeils. Willmanns Originalität ist eine viel bedauerlichere: sie besteht in dem fuir einen dentschen Professor unerhörten Ton, in der Respektlosigkeit vor dem Ernst der Wissenschaft. Und darin liegt das Gefährliche, das Ultramontane, dass er durch die Art, wie er seine Gründe vorbringt, wirken will, dass er in dem Leser den Glauben erzengen will, der Kampf gegen Kant wäre der Kampf gegen wissenschaftliche Unehrlichkeit und gegen protestantische Charakterlosigkeit. Denn natürlich, darauf läuft die Sache hinaus: die Wurzeln der Kantischen Philosophie liegen im Protestantismus; Luther hat den reinen Sinn zerstört, der sich pietătroll, 
ohne nach dem "Warum?" zu tragen, unter das Ewige beugt, das über dem Menschen waltet, und Kant hat für die freche Selbstüberhebung des Subjekts die philosophische, oder, wie Willmann stets von Kant sagt, die sophistische Formel gefunden. "Kant knüpft nicht an Luther an, weil sich näher liegende Quellen: die Aufklärung und der Rousseausche Individualismus darbieten, seiner Autonomie Nahrung zuzuführen; den Rückhalt beider Denkweisen aber bildet die Gesinnung, der Luther den klassischeu Ansdruck gegeben hatte. Er stellt das aller Verbindlichkeit enthobene [!] Subjekt der ganzen Welt gegenüber: „Ich soll meiner Seele raten, es ärgere sich denn die ganze oder halbe Welt." " (S. 401). Bei Luther war es die Freiheit des Christenmenschen, bei Kant ist es die Freiheit des sittlichen Menschen, die im Gegensatz zu den objektiven Mächten der katholischen Ethik das Individuum regieren soll. Kants Antonomismus war die Konsequenz von Luthers "Ich will meine Lehre ungerichtet haben von jedermann". Den historischen Zusammenhang hat Willmann sehr richtig hervorgehoben. Aber schon um dieses Zusammenhangs willen hasst er Kant, und noch mehr hasst er ihn wegen des mächtigen Aufschwungs, den in Folge der Befiuchtung mit Kantischen Ideen die protestantische Theologie in unserm Jahrhundert genommen hat. Indirekt richtet sich also zugleich gegen die wissenschaftliche protestantische Theologie Willmanns unwürdige Polemik; gegen sie soll der Katholizismas ansgespielt werden. -

Willmann spricht einmal (S. 504) von dem „konventionellen Respekt vor Kant". Dass sich der Denker ein eigenes Urteil anch gegenüber Kant erlaubt, das ist selbstverständlich. In der Wissenschaft soll es keinen konventionellen Respekt geben. Dass aber durch Entstellung der Kantischen Lehre der berechtigte Respekt vor seiner Grösse zu untergraben gesucht wird, das ist traurig. Willmann spricht allenthalben in seinem Buche von der „kantischen Sophistik“. Dass jeder Philosoph die Lehrmeinungen, mit denen er sich anseinandersetzt, auf ihre Richtigkeit prüft, dass er, wenn er Fehler gefunden hat, ihren psychologischen Grandlagen nachspürt, ist selbstverständlich. Dass aber durch eigenmächtige Verdrehung einer Lehre Sophismen hineingetragen und dann frohlokend aufgezeigt werden, das ist traurig. Seite 509 sagt Willmann: „Die Frage: was ist Wahrheit? nennt Kant eine Vexierfrage der Logiker, die , ungereimt ist und unnötige Antworten verlangt', so dass der Fragende und Antwortende, den belachenswerten Anblick gewähren, dass einer (wie die Alten sagten) den Bock melkt, der andere ein Sieb unterhält' (W. W. Hartenst.2 III, S. 86). Von einem Kant können wir nichts anderes erwarten, als dass er die Frage, die für die echten Denker den Mittelpunkt ihres Sinnens, Forschens und Lebens bildet, mit einem kynischen Witze abthut." Dass man, wenn man eine einzelne Stelle angreift, diese zitiert, ist selbstrerständlich. Dass man aber im Vertracen darauf zitiert, dass der Leser den Zusammenhang der Stelle weder im Gedächtnis hat, noch ihn nachschlägt, das ist traurig: Kant erklärt a. a. O. ganz ausführlich, warum der Begriff eines Kriteriums der Wahrheit der Erkenntnis der Materie nach in sich selbst widersprechend ist. Von nit einem Witze abthun ${ }^{4}$ ist also gar keine Rede. - Wollte man den einzelnen Unterstellungen, die sich Willmann gegen Kant zu 
Schulden kommen lässt, nachgehen, so könnte man Bände fullen. Fine Widerlegung nimmt ja immer mehr Raum ein als eine leichtfertig hingestellte Behauptung. Und thatsächlich ist das ganze Gericht, das der Prager Philosoph über Kant abhält, ein Ketzergericht. Kant soll verurteilt werden. Das Werturteil, dass Kant nichts wert sei, fungiert dabei als synthetisches Urteil a priori: es geht vor aller Erfahrung als eine ewige, zeitlose Wahrheit vorher and macht alle Erfahrung, wie sie nämlich Willmann brancht, erst möglich. Nur einige wenige Einwendungen von prinzipieller Wichtigkeit seien eingehend behandelt. Sie werden gentugen, um die Willmannsche Polemik za charakterisieren. Selbstverständlich ist unter der grossen Zahl der erhobenen Einwände auch mancher berechtigte. Neu ist fast gar nichts als der Ton, die unnachahmliche Stimmung, die Willmann seinem Erzengnis mitgiebt, und die im Leser jenen stolzen Zorn hervorruft, der vergebens sucht, das Wort „er weiss nicht, was er thut" zu finden. Gardeil hat diese Entschuldigung. Willmann hat sie nicht. Denn Willmann muss Kant kennen und Willmann muss fähig sein, ihn zu verstehen: das beweisen seine früheren Werke, die ihm mit Recht einen geachteten Namen verschafft hatten. Dass er aber diese "Geschichte des Idealismus" geschrieben hat - das ist traurig.

Wie Gardeil hält auch Willmann für nötig, zunächst das in der theoretischen Philosophie Kants gelegte Fundament zu untergraben, das freilich, auch wenn sich keine autonome Moral darauf gründen würde, schon ob seiner Gegnerschaft gegen dogmatische Metaphysik dem Thomisten unbequem sein musste. Er setzt ein bei der transscendentalen Aesthetik, speziell bei Kants Stellung zur Möglichkeit der Geometrie. Ein längeres Zitat aus der Kr. d. r. V., in dem Kant das Verfahren des Geometers mit dem des Philosophen vergleicht, wird richtig dahin zusammengefasst: „Das Geheimnis des' Erfolges des Geometers sieht Kant darin, dass er nicht auf das sieht, was er in seinem Begriffe vom Dreiecke wirklich denkt, sondern über ihn zu Eigenschaften, die in diesem Begriffe nicht liegen, hinausgeht, indem er seinen Gegenstand nach den Bestimmungen der reinen Anschaung bestimmt" (S. 387). Hören wir nun die Kritik, die Willmann hieran übt. "Kant überschätzt das rüstige Zugreifen des Geometers ansserordentlich; der Kunstgriff der euklidischen Demonstration, den dieser anwendet, beweist allerdings den Satz, aber künstlich und minder gat [!] als der Philosoph es kann, der nar das Wesen des Dreiecks ins Auge fasst. Aus seinem Wesen folgt, dass das Dreieck die Hälfte eines Parallelogramms gleicher Grundlinien und Höhe ist; in einem solchen aber ist die Winkelsumme der des Rechtecks gleich, mithin vier Rechte betragend, woron also anf die Hälfte, das Dreieck, deren zwei kommen." An späterer Stelle (S. 410) werden wir noch belehrt, dass die ganze transscendentale Hauptfrage "Wie sind synthetische Urteile a priori möglich ?" dahin zn beantworten ist: „Erweiterte, also synthetisch Zuwachs gewährende Erkenntnis einer Sache ohne neuerliche Erfahrungen darüber können wir durch Eindringen in deren Wesen gewinnen, in das wir durch Untersuchung seines Begriffes einzublicken vermögen." Schön! Halten wir uns an das Beispiel aus der Geometrie. Wie Willmann aus dem 
Wesen des Dreiecks ableiten will, dass es die Hälfte eines Parallelogramms gleicher Grundlinie and Höhe ist, und wie er aus dem Wesen des Parallelogramms ableiten will, dass seine Winkelsumme der des Rechtecks gleich ist, das mag er vor seinem logischen und mathematischen Gewissen verantworten. Selbst wenn er es aber könnte, so wäre noch immer der Schluss falsch, dass auf das Dreieck als die Häfte des Parallelogramms auch die Hälfte der Winkelsumme fallen muss. Mit gleichem Unrecht könnte er z. B. aus dem Wesen eines durch die Diagonalen in vier Dreiecke geteilten Quadrates folgern, dass auf jedes Dreieck als den vierten Teil der ganzen Figur die Winkelsumme von einem Rechten fällt. Für das gleichseitige Dreieck könnte er aus dem Wesen des Sechsecks nach gleicher Methode ableiten, dass seine Winkelsumme $120^{\circ}$ beträgt. Aehnliche Beispiele von Erkenntnis aus dem "Wesen" finden sich nun in jeder Metaphysik, und es ist kein kleines Verdienst Kants, dadurch dass er die synthetischen Urteile apriori der Metaphysik denen der reinen Mathematik und der reinen Naturwissenschaft gegenüber gestellt hat, gezeigt zn haben, dass den ersteren das fehlt, das allein den letzteren ihre Giltigkeit garantiert: die Möglichkeit einer transscendentalen Deduktion. Obige Beispiele dürften zum Beweise genügen, dass er nicht mit Unrecht , in der Heranziehung der Anschaunng “, wie ihm Willmann (S. 368) vorwirft, "das Entscheidende" gesehen hat. Es ist sehr gut, dass die Mathematik die Möglichkeit gewährt, mittels des gering geachteten „Kunstgrifts der euklidischen Demonstration" zu kontrolieren, welchen Wahrheitswert die Erkenntnis aus dem "Wesen" hat. Will Willmann einwenden, in den Beispielen von Quadrat and Sechseck wäre das "Wesen" nicht richtig erfasst, so mag er erklären, wie er es, ohne der Anschanung "das Entscheidende " za überlassen, besser kann: so lange er die Erklärung nicht gegeben hat, bleibt der. Satz in Geltung, dass sich das Wesen einer geometrischen Figur nur durch Konstruktion in der Anschaung erfassen lässt; unter "Wesen" aber kann vernünftigerweise nichts anderes verstanden werden als die Eigenschaften, die sich aus dem anschaulich gegebenen Objekt ableiten lassen. Das Erfassen des Wesens ist also das Posterins; das Prius ist immer die Anschaunng. Welche erkenntnis-

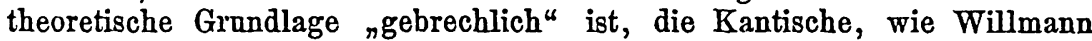
will (S. 412), oder die metaphysische Willmannsche; wessen Vorgehen "tumnltuarisch" ist (S. 412), das aus tiefem Verständnis der Mathematik geflossene Kantische oder das aus dem „Wesen ${ }^{\prime}$ argumentierende Willmannsche; wer vom „Taumel" (S. 413) erfasst ist, der Kritiker, oder sein Metakritiker; wer von beiden endlich der "Sophist" ist - das brancht nach dieser Probe kanm weiter diskutiert $\mathbf{z n}$ werden. Ich würde mich übrigens schämen, Ausdrücke wie ${ }^{\nabla} \nabla 0 \mathrm{~m}$ Taumel erfasst sein $^{\prime}$ gegen Willmann zu gebrauchen, wenn ich mich nicht durch sein Buch hierzu legitimiort wüsste.

Die unerquickliche Aufgabe, sich mit den auf solcher Erkenntnislehre erwachsenen Argumenten gegen die theoretische Philosophie im einzelnen abzufinden, sei jedem Leser überlassen. Vor der Besprechung von Willmanns moralphilosophischen Gegenargumenten. sei nur noch ein Wort aus seiner Kritik der transscendentalen Ideen zitiert. Kommentar ist 
unnötig, denn es spricht für sich selbst. „Wirkliche, ehrwürdige Lehrer des Menschengeschlechts waren es gewesen, die von Gott, Kosmos und Seele gesprochen hatten; diese Ideen sind alter als die Pyramiden and schon mancher Bube hatte mit Steinen nach ihnen geworfen. Auch Protagoras hatte erklärt: ,Von den Göttern bin ich nicht in der Lage za wissen, ob sie sind, oder ob sie nicht sind, denn vieles hindert solches zu wissen: die Dunkelheit des Gegenstandes und die Ktirze des Menschenlebens'; er hatte wenigstens offen gelassen, dass die vereinigte Arbeit vieler Menschenleben in der Frage weiter kommen könne, eine Bescheidnng, welche dem Vernunftkritiker fern liegt. Den Kosmos hatte Demokrit in einen Haufen von Atome verwandelt, aber er hatte wenigstens ihre Zahlen und Formen als objektiv gelten lassen, und seine i $\delta^{\varepsilon} \varepsilon \iota$ haben mehr Realgehalt, als die Kantischen Ideen. Die Seele hatten die Materialisten aller Zeit gelengnet, aber sich nicht erkühnt, sie als eine notwendige Fiktion nachzaweisen, wodnuch die Vernunftkritik nunmehr ihr Werk vollendete" (S. 425).

Und nun zur Ethik. „Das göttliche Gesetz bindet notwendig und allgemein und erwächst ans keiner Materie des Wollens, entspricht also dem, was Kant vom Gesetze verlangt, und der sich ihm konformierende Wille ist gut und doch nicht heteronom" (S. 465). Sehr schön! Aber warum konformiert sich denn der Wille dem göttlichen Gesetz? Warum erkennt er es als notwendig und allgemein bindend? Weil es Gott will? Dann gerät Willmann in die bekannte katholische Diallele. Da er jedoch als geschulter Logiker Diallelen verschmähen dürfte, wird er vielleicht antworten, dass ihm sein sittliches Bewusstsein die Richtigkeit der geoffenbarten Normen beglaubigt. Dann aber mag er die Worte, mit denen er im Texte fortfährt, auf sich selber beziehen: „Dem gegenüber statuiert nun Kant das autonome Prinzip mit der ihm eigenen Gewaltsamkeit und Sophistik. ,Selbst der Heilige des Evangelii muss zuvor mit unserem Ideal der sittlichen Vollkommenheit verglichen werden, ehe man ihn dafür erkennt ... Das heisst also: Unser Wille statuiert das Gute, unsere Vernunft malt sich ein Ideal desselben aus und dieses ist zugleich der Farbentopf, mit dem ein metaphysisches Ideal von Vollkommenheit hergestellt wird" (S. 465/6). Doch Willmann thut, als wolle er auf die Frage, warum er das göttliche Gesetz anerkennt, selber antworten: „Eines der stärksten Stücke der Kantischen Sophistik ist der Nachweis, dass die Aufstellung des göttlichen Gesetzes als Moralprinzip in dieselbe Kategorie gehört wie das Lustprinzip Epikurs" (S. 466). Es folgt nun eine Darlegung der bekannten Kantischen Begriindnng. Hierauf fährt Willmann fort: „So ergiebt sich denn Kant die Tabelle, in welcher er , die materialen Bestimmungsgründe im Prinzip der Sittlichkeit' zusammenfasst: I. subjektive, 1. äussere: a) der Erziehung (nach Montaigne), b) der bürgerlichen Verfassung (nach Mandeville), 2. innere: a) des physischen Gefühls (nach Epikur), b) des moralischen Gefühls (nach Hutcheson), II. objektive, 1. innere: der Vollkommenheit (nach Wolff und den Stoikern), 2. äussere: des Willens Gottes (nach Crusius and anderen theologischen Moralisten). Dass hinter Crasius, dem matigen Leipziger Theologen, der in jener Zeit das Gesetz Gottes als 
Moralprinzip hinzustellen wagte, die ganze Christenheit steht, die von diesem Prinzip siebzehn Jahrhanderte gelebt hatte, macht Kant nicht das geringste Bedenken bei dieser Zusammenstellnng; ebensowenig Epikurs und Wolffs Prinzipien die Innerlichkeit, also einen Vorzug vor dem christlichen zuzusprechen, welches somit neben Mandevilles Prinzip seine Stelle erhält." Und nun - kein Wort mehr hierüber. Kant ist widerlegt. $\mathrm{Ob}$ aber eine Widerlegang, die, statt Argumente zu bringen, drei Kreaze schlägt, eines dentschen Professors der Philosophie würdig ist, ist wohl einigermassen zweifelhaft. - Wie der Leser auf Grund der bereits gebrachten Zitate schon erwarten wird, wird an Stelle der Kantischen Lehre die Karikatur der Nietzscheschen Herrenmoral vorgeführt, dann aber behauptet, es wäre dies „das Kantische Riesengebide des Uebermenschen, wenn wir [d. h. Willmann] diesen Ausdrack des modernsten Autonomismus antizipieren dürfen: Ich bin mir selbst Gesetz; kein Herr über mir, nar in mir; soll ich gehorchen, so muss ich mir dabei selbst befehlen; habe ich Pflichten, so muss ich mir sie selbst anflegen; die Sittlichkeit ist eine Sache, die jeder mit sich selbst abzumachen hat; der Pflichterfüllende zahlt nur Schnlden an sich selbst, der Tugendheld ist, jenseits von gut und böse', denn sein Wille hat die Befugnis, beides zu stempeln. Das Wort Eritis sicnt dii, scientes bonum et malum, wird noch überboten, denn hier heisst es: statuentes bonum et malum und nicht einmal die Mehrzahl ist an der Stelle; jeder für sich ist statuens, nicht bloss ein Selbstherrscher in der sittlichen Welt, sondern selbst eine solche Welt, zum Makrokosmos aufgebläht" (S. 468/9). Diese Melodie wird lange fortgeblasen. Noch 10 Seiten weiter unten ist zu lesen: „Der Autonomismus ist seiner Natur nach Egoismus, kann also der selbstischen Neigungen nicht Herr werden, da er sie vielmehr auf den Thron setzt..." (S. 378). Nan kommt die nicht mehr verwundernde Anzweiflung von Kants Ehrlichkeit, aus der später (vgl. S. 487) die offene Behauptung wird, dass Kants Postulatenlehre nicht ernst gemeint war, und dann eine Zusammenfassung der Kritik der Kantischen Ethik, von der nur zwei Sätze genannt sein sollen: "Kants Moral ist in Wahrheit potenzierter Endämonismus, der sich die Larve der Rigorosität vorhält. - Es ist eine seltsame Figur, dieser kantische Tugendheld mit der schneidigen Skepsis, der titanische Biedermann, der mit allem aufgeräumt hat, der moralisierende Anarchist, die inkarnierte Pflichterfüllung mit dem Protest gegen jede Verpllichtung in der Tasche, der Uebermensch mit dem Zopfe" (S. 483). Ebenso wie diese Stelle mag das Urteil über Kants bekannte Lehre vom Gebet, von der allerdings klar ist, dass ihr der Vertreter der Rosenkranzkonfession wenig Geschmack abgewinnen kann, hier Platz finden: "Die ganze Hoffart, Verlogenheit and Heuchelei der Aufklärer spricht ans diesen Worten [sc. dem Zitat ans Kant], die zugleich ein grelles Schlaglicht auf die Ursachen der sozialen Dekomposition des protestantischen Deutschlands [!] werfen" (S. 492). Anch hier vermisst man natürlich wieder jedes sachliche Eingehen anf Kants Argumente: sie sind an und für sich schlecht; sie vertragen nicht, neben die katholische Lehre gestellt zu werden; ergo ist Kant verurteilt. Dieses Verfahren aber ist „tumultuarisch ${ }^{4}$, es ist das Verfahren des Hohepriesters, der mit den Worten „Er hat 
Gott gelüstert; was bedurfen wir weiteren Zeugnisses?" ${ }^{\mu}$ sein Kleid zerreisst.

Vorliegende Ausführungen haben absichtlich Willmann bei seinem Kampf um die Sittlichkeit verhältnismässig oft $\mathrm{zu}$ Wort kommen lassen. Denn gerade hier, in der kontruiren Auffassung des ethischen Prinzips, liegt der tiefste Grund für die Feindschaft, die gesetzt ist zwischen Kant und dem orthodoxen Ultramontanismus. Dieser sieht das Ziel des Menschen in dem gläubigen Erfassen der ein für alle Mal giltigen Glaubensinhalte. Was geglaubt werden soll, entscheidet die Kirche, und der einzelne hat weiter nichts za thun, als eben zn glauben. Das Recht freier Prüfung ist ihm genommen; entschädigt wird el dafür durch die Zusicherung, dass seine Kirche die allein seligmachende ist, nnd durch den Hinweis daranf, dass das, was er glauben soll, ja durchaus der Wahrheit entspricht. Dass eine solche Veräusserlichung des Glaubens unchristlich ist, hat Luther gezeigt; ihm gebührt das Verdienst, dem Individuum das Recht gegeben zu haben, nach dem Grund seines Glanbens zu fragen. Aber der Grund ist immer noch ein objektiver: er ist festgelegt in der heiligen Schrift, und das Recht des Subjekts geht nicht weiter als bis zar Frage, wie weit die von aussen dargebotene Auslegung schriftgemäss ist. Es ist klar, dass in der Konsequenz der Lutherschen Geistesthat die weitere Subjektivierung des religiösen Glaubens und der moralischen Ueberzeugung liegt. Es mussten dic Fragen auftauchen: Warnm soll ich überhaupt glauben? warum sittlich handeln? Und die Antwort konnte nur aus dem Subjekt und seiner inneren Erfalrung genommen werden. Der heutigen wissenschaftlichen Theologie sind die Fragen gelänfig, und sie hat sie gelernt von Kant. Kant seinerseits ist in der Subjektivierung von Religion und Moral weiter gegangen, als die Theologie je wird gehen können: der Philosoph ist freier, er darf, ja er muss seine Prinzipien vertreten ohne Rücksicht auf eventuelle Unzuträglichkeiten, die sich bei Anwendung auf eine Massenpsychologie ergeben könnten. Darum aber wird das ethische Prinzip der Autonomie des sittlichen Bewusstseins stets die Norm aller echten Sittlichkeit hleiben, und die Entwicklung der wissenschaftlichen theologischen Ethik dürfte sich der Weiterbildung der Kantischen Lehre asymptotisch nähern. - Kant hat zu den echten Weisen gehört, deren Leben mit ihrer Lehre harmonierte. Wenn Willmann sagt, es wäre ihm nicht Ernst mit seiner Religionslehre gewesen, wenn er von seinem „widerwärtigen, verlarvten Atheismus" (S. 495) spricht, so - sagt das eben Willmann. - Der Philosoph, der nicht von dogmatischen Voraussetzungen ausgeht, sondern jedem wissenschaftlichen Angriffsobjekt kritisch gegeniibersteht, weiss, wie viel menschliche Unvollkommenheit einer jeden Vernunftbethätigung anhaftet: er wird kritisch und skeptisch gegen alle Resultate, auch gegen die eigenen. Diese Skepsis ist der notwendige Ausfluss der Ehrlichkeit gegen sich selbst. Auch Kants Leben und Schriften sind Beweise zu entnehmen, dass in ihm jene höchste Form der Ehrlichkeit zum Durchbruch gekommen war. Alle die Gründe, die Willmann anführt, um zu zeigen, dass Kant seine praktischen Postulate für ,nützliche Selbstsuggestionen“ (S. 483) gehalten habe, gehören hierher. Willmann deklamiert: "Wenn Kant selbst seinen Rigorismas verspottete, 
so spricht sich darin eine Ahnung aus, wie wenig Berechtigung er zu rigorosem Stirnrunzeln hatte" (S. 483). Demgegenüber muss betont werden, dass die Ironie über sioh selbst bei keinem echten Philosophen ganz fehlen darf. In den "Sämtlichen Werken" dürfte man vielleicht bei manchem Denker vergebens nach ihr suchen. Dahin gehört derartiges auch nur in besonderen Fällen. Hingegen im persönlichen Leben kann es bei keinem ausbleiben, der es er'vst genug nimmt, $d$. $h$. der sich nicht auf Dogmata versteift, die unverrückbar feststehen, und zu diesen jeden seiner Lehrsätze zühlt. Der echte Philosoph ist nie fertig mit seiner Lehre, und eben weil er immer an ihr arbeitet, kann es geschehen, dass er eine Frage heute anders beantwortet als gestern, und dass er morgen wieder zar alten Antwort zurückkehrt. In seine Bücher wird er freilich so wenig wie möglich von diesem Kampf der Motive einfliessen lassen; hier soll eine Meinung zum Durchbrach kommen. Wenn sie aber mit der Niederschrift warten wollten, bis der Kampf absolut entschieden ist, so kämen gerade die tiefsten Denker nie zum Schreiben. - Mit fertigen Resultaten, die im Glanz ihrer Objektivität strahlen, und an denen nicht gerüttelt werden darf, will der Katholizismus die Menschheit beglücken; er ist "die Kirche, welche, vermöge ihres Anschlusses an das Ausserzeitliche und dessen Güter, die Wirren und Gefahren der Zeit zu überblicken und Hülfe zu bringen vermag“ (S. 960). Anders der protestantische Idealismns. Er hat seinen Angelpunlst gefunden im Werte des unendlichen Strebens. Er sucht das Ewige, sein Ringen ist seine Sittlichkeit. „Nicht die Wahrheit, in deren Besitz irgend ein Mensch ist, sondern die aufrichtige Mühe, die er angewandt hat, hinter die Wahrheit zu kommen, macht den Wert des Menschen. Denn nicht durch den Besitz, sondern durch die Nachforschung der Wahrheit erweitern sich seine Kräfte, worin allein seine immer wachsende Vollkommenheit besteht."

Gerade, als ich mit diesem Wort Lessings meine Ausführungen zu schliessen dachte, kam mir Paulsens Artikel „Das jüngste Ketzergericht über die moderne Philosophie" (Dentsche Rundschau XXIV, 11), gleichfalls ein Protest gegen das Willmannsche Buch, in die Hand. Ich kann mir nicht versagen, den geistreichen Erörterungen eine Stelle zu entnelımen, die mir die Psychologie des nltramontanen Standpunktes aufzuhellen sehr geeignet scheint: "Wer sich entschlossen hat, für sich auf das Recht des freien Denkens, $d . h$. auf das Urrecht des Geistes, sich im Denken allein durch Gründe der Vernunft bestimmen zu lassen, zu verzichten, der wird dann geneigt sein, bei Andern das freie Denken als verwerflichen Hochmut zu verdammen. Und er wird den freien Denker hassen, weil er ihn als bedrohlich für seine Sicherheit, ja sein blosses Dasein als Vorwurf empfindet" (a. a. O. S. 195). Damit ist der Einleitungsgedanke wieder erreicht: Willmanns Buch ist die Parodie auf die Behauptung, dass der Katholizismus je Prinzip des Fortschritts sein könne. Freilich behauptet Schell, auch der Katholizismus lasse dem Individuum das Recht freier Prüfung in Gewissenssachen. Wohin aber führt solche Prüfung bei gleichzeitiger Anerkennnng des Autoritätsprinzips? Gar viel ,Katholisches Allzukatholisches' bei Schell selbst giebt dic Antwort, 